Published in final edited form as:

Gastroenterology. 2015 May ; 148(5): 967-977.e2. doi:10.1053/j.gastro.2015.01.032.

\title{
Decreasing Mortality Among Patients Hospitalized With Cirrhosis in the United States From 2002 Through 2010
}

\author{
Monica L. Schmidt ${ }^{1}$, A. Sidney Barritt ${ }^{2}$, Eric S. Oman ${ }^{3}$, and Paul H. Hayashi ${ }^{2}$ \\ 1 University of North Carolina Liver Center and Gillings School of Global Public Health \\ ${ }^{2}$ Division of Gastroenterology and Hepatology, University of North Carolina, Chapel Hill, North \\ Carolina \\ ${ }^{3}$ Division of Gastroenterology and Hepatology, Indiana University, Indianapolis, Indiana
}

\begin{abstract}
BACKGROUND \& AIMS-It is not clear whether evidence-based recommendations for inpatient care of patients with cirrhosis are implemented widely or are effective in the community. We investigated changes in inpatient outcomes and associated features over time.

METHODS—By using the Healthcare Cost and Utilization Project, National Inpatient Sample, we analyzed 781,515 hospitalizations of patients with cirrhosis from 2002 through 2010 . We compared data with those from equal numbers of hospitalizations of patients without cirrhosis and patients with congestive heart failure (CHF), matched for age, sex, and year of discharge. The primary outcome was a change in discharge status over time. Factors associated with outcomes were analyzed by Poisson modeling.
\end{abstract}

RESULTS-The mortality of patients with and without cirrhosis, and patients with CHF, decreased over time. The absolute decrease was significantly greater for patients with cirrhosis (from $9.1 \%$ to $5.4 \%$ ) than for patients without cirrhosis (from $2.6 \%$ to $2.1 \%$ ) or patients with CHF (from $2.5 \%$ to $1.4 \%)(P<.01)$. However, relative decreases were similar for patients with cirrhosis $(41 \%)$ and patients with CHF (44\%). For patients with cirrhosis, the independent mortality risk ratio decreased steadily to 0.50 by 2010 (95\% confidence interval, $0.48-0.52$ ), despite patients' increasing age and comorbidities. Hepatorenal syndrome, hepatocellular carcinoma, variceal bleeding, and spontaneous bacterial peritonitis were associated with a higher mortality rate, but the independent mortality risks for each decreased steadily. Sepsis was associated strongly with increased mortality, and the risk increased over time.

(C) 2015 by the AGA Institute

Reprint requests, Address requests for reprints to: Paul H. Hayashi, MD, MPH, Division of Gastroenterology and Hepatology, University of North Carolina, Burnett-Womack Building, Room 8011, Chapel Hill, North Carolina 27599. paul_hayashi@med.unc.edu.

Supplementary Material

Note: To access the supplementary material accompanying this article, visit the online version of Gastroenterology at http:// dx.doi.org/10.1053/j.gastro.2015.01.032.

Conflicts of interest

The authors disclose no conflicts. 
CONCLUSIONS-Among patients with cirrhosis in the United States, inpatient mortality decreased steadily from 2002 through 2010, despite increases in patient age and medical complexity. Improvements in cirrhosis care may have contributed to increases in patient survival beyond those attributable to general improvements in inpatient care. Further improvements might require an increased use of proven therapies and the development of new treatments-particularly for sepsis.

\section{Keywords}

Liver Failure; Renal Failure; Decompensation; Predictors

Cirrhosis is the eighth leading cause of death and years of life lost in the United States. ${ }^{1}$ The course of cirrhotic liver failure often requires hospitalizations for complications such as renal failure, variceal bleeding, ascites, hepatic encephalopathy, and hepatocellular carcinoma. In addition, cirrhosis affects the outcome of non-liver-related illnesses requiring hospitalization. Care of cirrhosis patients is complex and often is managed by a team of specialists including gastroenterologists, hepatologists, intensivists, and nephrologists. The risk of mortality can be high, but careful management can mitigate this risk. ${ }^{2-5}$ Over the past 10 to 15 years, significant advancements have been made in the management of hepatorenal syndrome (HRS), variceal bleeding, spontaneous bacterial peritonitis (SBP), ascites, and hepatocellular carcinoma (HCC) ${ }^{6-9}$ Such advances have led to the dissemination of several evidence-based practice guidelines by all 3 major hepatology associations. ${ }^{10-12}$ Cirrhotic patients also may benefit from non-liver-specific guidelines such as sepsis care, particularly early identification of the systemic inflammatory response syndrome, and antibiotic administration. ${ }^{13}$

Studies have indicated that guideline dissemination and implementation are effective in changing practice behaviors and improving patient outcomes. ${ }^{14,15}$ Therefore, we hypothesized that guidelines for inpatient cirrhosis care have penetrated the wider medical community and have resulted in better outcomes. We analyzed a large, nationally representative sample of cirrhosis patients who were hospitalized from 2002 through 2010 across the United States. Our goal was to see if inpatient mortality of cirrhotic patients has improved over time and to evaluate clinical variables associated with mortality including specific cirrhosis-related diagnoses and interventions.

\section{Materials and Methods}

\section{Data Source}

Data were extracted from discharges in the Nationwide Inpatient Sample (NIS), Healthcare Cost and Utilization Project (HCUP), Agency for Healthcare Research and Quality for the years 2002-2010. ${ }^{16}$ The HCUP NIS is a 20\% stratified sample of hospitals in the United States. It is the largest all-payer inpatient care database with hospitals spread across 46 states, and these states comprise more than $97 \%$ of the US population. The HCUP NIS contains more than 8 million hospital stays per year from more than 1000 hospitals. A full range of hospitals is sampled including community and academic centers. Data are entered as individual discharge records. Each record has a unique identifier, demographic data, 
hospital type, admission type, transfer status, hospitalized inpatient mortality indicator, discharge to palliative care, primary diagnosis, secondary diagnoses (up to 15), and procedure codes (up to 15). Admission diagnoses are not included in this data set. Time from admission to various primary procedures (eg, endoscopy and paracentesis) is provided as well as a diagnosis and procedure groups and patient comorbidity elements. Third-party payer status is included in the data set, however, "self-pay" is not defined further (ie, paid out of pocket vs inability to pay).

Cohort selection-The HCUP NIS contains 71,718,458 individual discharge records from 2002 through 2010. By using the International Classification of Diseases, 9th revision, Clinical Modification (ICD-9-CM) diagnostic and procedural codes, we extracted the subpopulation of patient admissions with cirrhosis $(n=781,678)$ from the HCUP NIS database (Supplementary Table 1). We included hospital discharges from 2002 through 2010 that had one or more of the following diagnoses: alcoholic cirrhosis of liver (571.2), biliary cirrhosis (571.6), or cirrhosis without mention of alcohol (571.5). Identification of cirrhotic patients using these codes in administrative data from the Veterans Affairs had a positive predictive value of $90 \%$ and a negative predictive value of $87 \% .{ }^{17}$ We included elective admissions $(n=57,960)$.

To determine whether changes in mortality were specific to cirrhosis care, we compared mortality data between the cirrhotic cohort and a noncirrhotic (NC) cohort matched 1:1 on age, sex, and year of discharge. Inpatient care of congestive heart failure also has advanced. Therefore, we compared mortality data between the cirrhotic cohort and a noncirrhotic cohort with congestive heart failure (CHF), matched 1:1 on age, sex, and year of discharge. After matching, each cohort contained 781,515 hospitalizations. These cohorts became the focus of our analyses.

Outcomes, primary variables, and covariates-Our primary outcome was "died while hospitalized," as labeled in the HCUP NIS. The secondary outcome was discharge to palliative care. Our primary independent variable was year of discharge. Primary covariates of interest were 5 cirrhosis-related diagnoses, which can be fatal and require specific interventions: HRS, HCC, variceal bleeding, sepsis, and SBP. We also examined 3 procedures: esophagogastroduodenoscopy (EGD) within 1 day of admission, paracentesis within 1 day of admission, and transvenous intrahepatic portosystemic shunt (TIPS) at any time during the stay. These are captured readily in the HCUP NIS. Unfortunately, many other important cirrhosis-related interventions and diagnostic tests (eg, albumin use for SBP) are not captured and therefore were not available for analysis. Twenty-eight of the Agency for Healthcare Research and Quality comorbidity measures contained in the HCUP NIS were combined to generate the Elixhauser Comorbidity Index. ${ }^{18,19}$ Use of this index to adjust for comorbidities in administrative databases was supported by Austin et al ${ }^{19}$ using mathematic proofs. Major diagnoses and predictive covariates were defined using ICD-9CM codes and are shown in Supplementary Table 1. To define the time to paracentesis or EGD, the HCUP NIS data element indicating the day on which the procedure was performed was used in conjunction with the primary procedure. If EGD or paracentesis was performed within 1 day after admission, we captured it using a combination of a time-to-procedure 
code and our primary diagnosis codes. ${ }^{16}$ We also adjusted for a length of stay less than 2 days because many of these hospitalizations may represent the extremes of mortality risk that are less influenced by inpatient care (eg, moribund status, false alarm admissions for spurious laboratory results, and overnight observations after minor procedures). Patient demographic (eg, age, sex) and hospital characteristics (eg, academic, community based) were extracted from the HCUP NIS and included in the analysis.

Statistical analyses-Data were analyzed using the Stata 12.0 software package (Stata Corp LP, College Station, TX). HCUP NIS data are provided in a 2-stage cluster design incorporating clustering at the hospital level and discharge level. HCUP provides weighting of discharges based on the hospital type and volume of discharges relative to their sampling region. Two-way chi-square analyses were performed on categoric variables and $t$ tests were performed for continuous variables. Poisson regression with robust (Huber-White) standard errors was used to determine incident risk ratios (RR) for predictors of in-hospital mortality. ${ }^{20}$ We tested the Poisson models for overdispersion using a Pearson goodness-offit test. Models were not overdispersed $(P=1.00)$ and were appropriate for our analyses. Regression analyses controlled for several variables including calendar year of admission, major diagnostic and procedures covariates, age, sex, race, primary payer, Elixhauser comorbidity index, admission source, length of stay, and weekend admission. Referent categories were admission year 2002, age younger than 40 years, male sex, white, a routine admission, and self-pay listed as the primary payer.

We also hypothesized that an increased uptake of specific guidelines for the care of HRS, SBP, sepsis, variceal bleed, and HCC may have improved survival and would be reflected in decreasing mortality risk ratios from year to year. Therefore, we created interaction terms between each diagnosis and year of discharge (eg, HRS × 2002, HRS × 2003, and so forth). A more appropriate use of EGD within 1 day of admission, paracentesis within 1 day of admission, and TIPS may have led to similar decreasing risk ratios for inpatient mortality over time. To investigate this possibility, we created interaction terms between each of these interventions and year of discharge (eg, TIPS $\times 2002$, TIPS $\times 2003$, and so forth). We also examined EGD within a day, paracentesis within a day, and TIPS at any time during the hospitalization restricted to patients with a variceal bleed or ascites.

\section{Study Approval and Data Use Agreement}

The Institutional Review Board of the University of North Carolina at Chapel Hill approved the research protocol before beginning this research. A data use agreement was in place with the Agency for Healthcare Research and Quality for use of the HCUP NIS data.

\section{Results}

\section{Patients Characteristics and Univariate Analyses of In-Hospital Mortality}

Patient demographics for all 781,515 hospitalizations of patients with cirrhosis are presented in Table 1, along with the subgroups of hospitalizations ending in discharge without hospice care (713,537 [91.3\%]), death in the hospital (57,955 [7.4\%]), and discharge to hospice care $(10,023[1.3 \%])$. Although the number of cirrhosis hospitalizations increased over the years, 
the proportion of patients dying in the hospital decreased (Table 1). Alcohol-related liver disease was present in nearly half of all admissions, and ascites and encephalopathy were the most common cirrhosis-related complications. More than two thirds were admitted through the Emergency Department (ED) and slightly less than two thirds had Medicaid and/or Medicare coverage. Although 33\% had a diagnosis of ascites, only $9.2 \%$ had paracentesis on the first day of hospitalization.

Patients who died had a higher proportion of cirrhosis complications, especially HRS and sepsis. A higher proportion of patients who died were transferred from another hospital or were hospitalized for a short time ( $<2$ days). Patients discharged to hospice had a significantly higher prevalence of HCC, but a lower proportion of transfers from another hospital. Both increased age and comorbidities were more common in patients who died or were discharged to hospice, but skewing toward increased age and amount of comorbidities was even higher in the hospice subgroup (30.2\% older than age 70 and mean Elixhauser Comorbidity Index of 3.3). Several variables such as payer status, race, sex, and admission on a weekend were statistically different, but the differences were of unclear clinical significance.

\section{Trends Over Time}

Despite an increase in hospitalizations involving cirrhosis, inpatient mortality rates steadily decreased from $9.1 \%$ in 2002 to $5.4 \%$ in 2010 . The mortality rates for the noncirrhotic and the noncirrhotic, CHF cohorts decreased, but to a significantly lesser degree (Figure 1). However, the relative decrease in mortality was similar for cirrhosis and CHF patients (41\% and 44\% decreases, respectively), and was smallest for the noncirrhotic cohort (19\% decrease). The improvement in survival for cirrhosis patients occurred despite increasing age and comorbidities. There was steady increased skewing toward increased age brackets over time $(70.5 \%$ in age $>50 \mathrm{y}$ in 2002 , vs $77.9 \%$ in age $>50$ in 2010). The mean Elixhauser Comorbidity Index for cirrhosis patients was 2.5 in 2002 and steadily increased to 3.4 by $2010(P<.05)$. Nevertheless, inpatient mortality rates for cirrhosis patients decreased across all age brackets, even those older than ages 70 and 80 years (Figure 2). Length of stay also decreased steadily from a mean of 6.95 days in 2002 to 5.88 days in $2010(P<.001)$. Our secondary outcome, discharged to palliative care, was used infrequently but increased steadily by 4 -fold from $0.4 \%$ in 2002 to $1.7 \%$ in $2010(P<.05)$.

\section{Multivariate Analysis of In-Hospital Mortality and Discharge to Palliative Care}

By Poisson regression, each passing year was associated independently with decreasing risk (RR) of inpatient mortality $(P<.001)$ (Tables 2 and 3, Figure 3). By 2010, the risk of dying in the hospital had decreased by $50 \%$ (RR, 0.50; 95\% confidence interval [CI], 0.48-0.52) compared with 2002. As expected, increased age, sepsis, HRS, variceal bleed, and HCC all were associated independently with increased risk of death. The highest independent risks for death were seen with sepsis (RR, 4.70; 95\% CI, 4.61-4.79) and HRS (RR, 3.39; 95\% CI, 3.31-3.47). Starting the hospitalization as a transfer from another hospital increased the mortality risk by $60 \%$. Having some form of insurance or third-party payer was associated with a lower mortality rate compared with self-pay. Hospital type (eg, academic vs community based) was not associated with mortality risk (data not shown). Controlling for 
hospitalization for shorter than 1 day, instead of fewer than just 2 days, and excluding the $1.3 \%$ of cirrhosis patients discharged to palliative care, did not significantly change any of the incident risk ratios shown in Table 2. The noncirrhotic cohort with CHF had a similar $50 \%$ decrease in their mortality risk ratio, whereas the overall noncirrhotic comparison cohort had a 34\% decrease in risk (Table 2).

The mortality risk ratios for the interaction terms of cirrhosis-related diagnoses (HRS, SBP, variceal bleed, and HCC) by discharge year all decreased (Figure 3 and Supplementary Table 1), with the most remarkable decrease occurring for HRS. Thus, for each cirrhosis complication, except sepsis, the risk of dying was lower in 2010 compared with 2002. In contrast, the risk of dying for a cirrhosis patient with sepsis was $28 \%$ higher (RR, 3.62; 95\% CI, 3.32-3.95 in 2002; vs RR, 4.63; 95\% CI, 4.41-4.86 in 2010). The mortality risk for EGD within 1 day of admission, paracentesis within 1 day of admission, and TIPS also tended to decrease over time.

To further examine the effect of early EGD, early paracentesis, and TIPS, we reran the Poisson model restricting patients to those with variceal bleed or ascites. When we restricted the model to patients with ascites, paracentesis within 1 day of admission was associated independently with a $12 \%$ decrease in mortality risk (RR, 0.88 ; 95\% CI, 0.84-0.92).

However, when we restricted the model to patients with a variceal bleed, EGD within 1 day of admission was associated with a $22 \%$ increased risk of death (RR, 1.22; 95\% CI, 1.151.30). TIPS at any time during the hospitalization had a mortality RR of 1.17 (95\% CI, 1.081.27) when restricted to patients with ascites, and 1.94 (95\% CI, 1.79-2.11) when restricted to patients with variceal bleeding.

Multivariate Poisson modeling for our secondary outcome of discharge to palliative care (Table 3) showed similar associations compared with our primary outcome of mortality (Table 2), but there were notable differences. Risk ratios for discharge to palliative care increased from year-to-year, consistent with its increase in use. Although increasing age, HRS, and HCC all were associated independently with palliative care discharges, the association was smaller for HRS and larger for HCC when compared with their risks of mortality. Interestingly, sepsis, TIPS, length of stay fewer than 2 days, admission through the ED, and transfer from an outside facility carried lower risk ratios of being discharged to palliative care.

\section{Discussion}

In this large representative sample of US hospitalized patients with cirrhosis, we found reduced inpatient mortality rates from 2002 through 2010. The absolute rate of dying in the hospital decreased steadily by $41 \%$ from $9.1 \%$ in 2002 to $5.4 \%$ in 2010 (Figure 1 ). On multivariate analysis, the incident RR of mortality also decreased steadily by $50 \%$ (RR, $0.50 ; 95 \%$ CI, $0.48-0.53$ by 2010) (Table 2 ). The decrease in mortality rate for cirrhosis patients was significantly larger compared with NC patients (Figure 1 and Table 2). NC patients had only a $0.5 \%$ absolute decrease in mortality, representing a $19 \%$ relative decrease and a $34 \%$ decrease in risk of death on multivariate analysis (RR, 0.66). Therefore, 
the improvement in cirrhosis survival may be owing to better cirrhosis-specific care that extends beyond general improvements in inpatient care.

This consistent decrease in mortality rate and risk for cirrhosis patients, year to year, occurred despite increasing age and comorbidities. Indeed, the inpatient mortality rate decreased across all age groups (Figure 2). The steady decrease in mortality risk was independent of several cirrhosis-related diagnoses, payer status, sex, and race (Table 2), and persisted after excluding patients discharged to palliative care and patients hospitalized for only 1 or 2 days. Noncirrhotic CHF patients had a smaller absolute decrease in mortality rate from $2.5 \%$ to $1.4 \%$, but the relative decrease and change in mortality risk ( $40 \%$ and $50 \%$ decreases, respectively) were similar to patients with cirrhosis. Improving CHF care and outcomes have been suggested in other studies. ${ }^{21,22}$

A single-center study from the United States and a regional study from Spain also suggested improved survival for cirrhosis patients over time, but these studies had smaller cohorts, did not control for palliative care discharges, and did not compare outcomes with noncirrhotic or non-liver-related chronic disease cohorts. ${ }^{23,24}$ Our study, using the HCUP NIS, included a large cohort and spanned a nation. The HCUP NIS is a $20 \%$ sampling of US hospitals, stratified by several variables including region and hospital type. Strong associations between inpatient mortality and well-known risk factors such as increased age, sepsis, and HRS lend validity to our data and modeling (Table 2). Therefore, our findings of decreasing inpatient mortality and risk of death are well supported and representative of inpatient cirrhosis care across the United States.

Improvement in cirrhosis care may be contributing to this decrease in inpatient mortality rates. Several guidelines and reviews regarding the management of HRS, SBP, variceal bleed, and HCC have been published in the past 10-15 years. ${ }^{6,9,10,25-29}$ Interaction terms between these complications and year of discharge yielded a decrease in mortality RRs for each diagnosis. HRS and SBP had the largest percentage decreases in mortality risk (HRS: RR, 5.6, 95\% CI [5.6-6.0] to 3.2 [3.0-3.4]; SBP: RR, 1.9 [1.7-2.1] to 1.2 [1.1-1.3]), suggesting that patients admitted with either HRS or SBP had a roughly $40 \%$ better chance of survival in 2010 compared with 2002 (Figure 3, Supplementary Table 2). Over the past 10-15 years, the diagnosis and management of HRS has been clarified by the International Ascites Club, which may have led to earlier diagnoses and more use of albumin and vasoactive medications. ${ }^{30}$ Similarly, clear recommendations for early diagnostic paracentesis and use of albumin for SBP may be penetrating community practice., ${ }^{9,10}$

However, we were unable to link improved outcomes with the few cirrhosis-specific interventions available in this data set. As seen in a prior study using the HCUP NIS, ${ }^{31}$ paracentesis within 1 day of admission for patients with ascites was associated with a $12 \%$ mortality risk reduction among cirrhotic patients with ascites, but we did not see an increased use of early paracentesis over time. EGD within 1 day was associated with an increase in mortality among those with variceal bleeding, which runs counter to guidelines for early endoscopy. Early EGD probably is performed more commonly with severe and rapidly bleeding patients which creates a selection bias for patients with a higher mortality risk. Similarly, TIPS at any time during the hospitalization was associated with a higher 
mortality rate probably because it is used for more advanced portal hypertension. When restricted to variceal bleed patients, there was an even higher mortality risk, which reflects the increased acuity for patients failing endoscopic control of bleeding. Unfortunately, the HCUP NIS does not contain details on the many other cirrhosis care interventions that have evolved in the past 10-15 years. Uptake of these other evidence-based recommendations could explain the decreases in mortality risk. Indeed, it is difficult to explain such consistent decreases without some attribution to improvement in cirrhosis care.

The independent mortality risk for sepsis increased over time to surpass the risk related to HRS (Figure 3). It occurred despite the ongoing "surviving sepsis campaign." The reasons for this increase are unclear and are at odds with a recent study that also used the HCUP NIS, which suggests that overall sepsis mortality rates are decreasing. However, hepatic "end-organ failure" made up only 4\%-5\% of all sepsis patients and no cirrhosis-specific analyses were provided. Cirrhosis patients have particularly poor hemodynamic reserve, with wider perturbations in immune inflammatory and compensatory responses that could hinder survival. ${ }^{34}$ Therefore, it is possible that cirrhosis patients are doing much worse with sepsis compared with other patients. The surviving sepsis campaign may need guidelines that specifically target cirrhosis patients.

Although the use of palliative care discharges remains low, its increase $(0.4 \%-1.7 \%)$ is noteworthy. Patients discharged to palliative care tended not to be admitted by transfer from another hospital or through the ED (Table 3). Palliative care was associated strongly with increased age, hepatic decompensation, HCC, and comorbidities. Thus, the increase in palliative care probably represents an improvement in care. Unnecessary morbidity can be significant for advanced liver failure patients if palliative care is not implemented. In a Canadian study, where only $11 \%$ of cirrhotic patients deemed inappropriate for transplant received palliative care, $80 \%$ went on to be hospitalized, $50 \%$ received intensive care unit care, and 53\% died as inpatients. ${ }^{35}$ The investigators and the authors of an accompanying editorial argued that palliative care would have prevented suffering, and is woefully underutilized. ${ }^{36}$ We confirm low use in the United States, but also a 4-fold increase since 2002.

The HCUP NIS is limited by its inability to identify individual patients who are re-admitted. Therefore, the decrease in mortality rates for cirrhotic patients could be owing partly to increased re-admissions. If discharges are premature or poorly executed, leading to unnecessary readmissions or death at home, then any claim of improved quality of care is undermined. Indeed, the length of stay decreased from a mean of 6.95 days in 2002 to 5.88 days in $2010(P<.001)$. However, better inpatient care also leads to a shortened length of stay and increased re-admissions. Need for hospitalization increases as part of the dying process for many cirrhotic patients, particularly if hospitals become better at saving sicker cirrhotic patients year after year. Indeed, re-admission rates have been criticized as a poor quality indicator for just this reason. ${ }^{37}$ Overall, as few as $12 \%$ of re-admissions may be preventable, and lower inpatient mortality rates for heart failure patients has been associated with re-admissions because the sickest patients are kept alive only to be re-admitted. ${ }^{38,39} \mathrm{~A}$ similar relationship between better inpatient care and increased re-admissions would be particularly true for an aging cirrhotic population with increasing comorbidities. A single- 
center retrospective study showed a 37\%, 30-day re-admission rate for cirrhosis patients, and $78 \%$ were deemed unavoidable. ${ }^{40}$

As with any administrative data set, the accuracy and scope of data had limitations. ICD-9CM codes can be inaccurate, particularly for liver-related codes other than cirrhosis. ${ }^{41}$ However, our use of several combined liver-related diagnostic codes has been shown to have greater accuracy ${ }^{31,41-43}$ (Supplementary Table 1). We used several cirrhosis codes that have high positive (90\%) and negative (87\%) predictive values. ${ }^{17}$ Misclassification of discharge diagnoses was quite low in this data set. The 2011 HCUP NIS Data Quality Report indicated that only $0.06 \%$ of the discharge summaries checked against the American Hospital Association database were missing principle diagnoses, and only $0.06 \%$ had invalid diagnoses. The HCUP NIS does not capture many liver failure severity variables and laboratory results accurately (eg, model for end-stage liver disease and Child-Pugh scores). Although there is no reason to believe the severity of liver failure among hospitalized patients decreased over the years, we could not test for such an influence on improving survival. Finally, we cannot comment on longer-term (eg, 3 and 6 months), post-discharge outcomes with this data set.

Nonetheless, our study represents outcomes of a large sampling of hospitalized cirrhotic patients and spans a nation over nearly a decade. The improving inpatient survival despite aging and more medically complex cirrhotic patients is remarkably consistent across several cirrhosis complications and suggests improving cirrhosis care that may extend beyond general improvements in inpatient care. On the other hand, sepsis had an increasing mortality risk, suggesting that cirrhotic patients may need a more tailored approach to sepsis. Use of palliative care will continue to increase and likely reflects an appropriate transition for older and medically complex cirrhotic patients. As cirrhosis-related admissions continue to increase and hospitals increasingly are held accountable for outcomes, our data may help in setting appropriate quality care indicators, including guideline use, adjusted mortality risk, and use of palliative care.

\section{Supplementary Material}

Refer to Web version on PubMed Central for supplementary material.

\section{Acknowledgements}

The authors would like to thank Mark Holmes, PhD, Associate Professor, Health Policy and Management, Gillings School of Global Public Health, for advice on our Poisson modeling and analysis.

Funding

This work was supported in part by the National Institutes of Health (KL2 TR001106).

\section{Abbreviations used in this paper}

$\begin{array}{ll}\text { CI } & \text { confidence interval } \\ \text { CHF } & \text { congestive heart failure }\end{array}$


ED

EGD

$\mathrm{HCC}$

HCUP NIS

HRS

ICD-9-CM

NC

RR

SBP

TIPS emergency department

esophagogastroduodenoscopy

hepatocellular carcinoma

Healthcare Cost and Utilization Project, National Inpatient Sample

hepatorenal syndrome

International Classification of Diseases, 9th revision, Clinical Modification

noncirrhotic

risk ratio

spontaneous bacterial peritonitis

transvenous intrahepatic portosystemic shunt

\section{References}

1. US Burder of Disease Collaborators. The state of US health, 1990-2010: burden of diseases, injuries, and risk factors. JAMA. 2013; 310:591-608. [PubMed: 23842577]

2. Carbonell N, Pauwels A, Serfaty L, et al. Improved survival after variceal bleeding in patients with cirrhosis over the past two decades. Hepatology. 2004; 40:652-659. [PubMed: 15349904]

3. Weinstein MP, Iannini PB, Stratton CW, et al. Spontaneous bacterial peritonitis. A review of 28 cases with emphasis on improved survival and factors influencing prognosis. Am J Med. 1978; 64:592-598. [PubMed: 645725]

4. Chalasani N, Kahi C, Francois F, et al. Improved patient survival after acute variceal bleeding: a multicenter, cohort study. Am J Gastroenterol. 2003; 98:653-659. [PubMed: 12650802]

5. Fernandez J, Navasa M, Planas R, et al. Primary prophylaxis of spontaneous bacterial peritonitis delays hepatorenal syndrome and improves survival in cirrhosis. Gastroenterology. 2007; 133:818824. [PubMed: 17854593]

6. Garcia-Tsao G, Sanyal AJ, Grace ND, et al. Prevention and management of gastroesophageal varices and vari-ceal hemorrhage in cirrhosis. Hepatology. 2007; 46:922-938. [PubMed: 17879356]

7. Sherman M, Bruix J, Porayko M, et al. Screening for hepatocellular carcinoma: the rationale for the American Association for the Study of Liver Diseases recommendations. Hepatology. 2012; 56:793-796. [PubMed: 22689409]

8. Runyon BA;AASLD. Introduction to the revised American Association for the Study of Liver Diseases Practice Guideline management of adult patients with ascites due to cirrhosis 2012. Hepatology. 2013; 57:1651-1653. [PubMed: 23463403]

9. Sort P, Navasa M, Arroyo V, et al. Effect of intravenous albumin on renal impairment and mortality in patients with cirrhosis and spontaneous bacterial peritonitis. N Engl J Med. 1999; 341:403-409. [PubMed: 10432325]

10. Runyon BA, Committee APG. Management of adult patients with ascites due to cirrhosis: an update. Hep-atology. 2009; 49:2087-2107.

11. Management of ascites, spontaneous bacterial peritonitis, and hepatorenal syndrome in cirrhosis. EASL Clinical Practice Guidelines. 2010 Aug.2010(4) Available: http://www.easl.eu/_clinicalpractice-guideline.

12. Sarin SK, Kumar A, Almeida JA, et al. Acute-on-chronic liver failure: consensus recommendations of the Asian Pacific Association for the study of the liver (APASL). Hepatol Int. 2009; 3:269-282. [PubMed: 19669378]

13. Jones AE, Puskarich MA. The Surviving Sepsis Campaign guidelines 2012: update for emergency physicians. Ann Emerg Med. 2014; 63:35-47. [PubMed: 24067755] 
14. Grol R, Grimshaw J. From best evidence to best practice: effective implementation of change in patients' care. Lancet. 2003; 362:1225-1230. [PubMed: 14568747]

15. Grimshaw J, Eccles M, Tetroe J. Implementing clinical guidelines: current evidence and future implications. J Contin Educ Health Prof. 2004; 24(Suppl 1):S31-S37. [PubMed: 15712775]

16. HCUP Nationwide Inpatient Survey. [Accessed August 2011] Available: www.hcup-us.ahrq.gov/ nisoverview.jsp

17. Kramer JR, Davila JA, Miller ED, et al. The validity of viral hepatitis and chronic liver disease diagnoses in Veterans Affairs administrative databases. Aliment Pharmacol Ther. 2008; 27:274282. [PubMed: 17996017]

18. Elixhauser A, Steiner C, Harris DR, et al. Comorbidity measures for use with administrative data. Med Care. 1998; 36:8-27. [PubMed: 9431328]

19. Austin SR, Wong YN, Uzzo RG, et al. Why summary comorbidity measures such as the Charlson Comorbidity Index and Elixhauser score work. Med Care 2013 Epub ahead of print.

20. Zou G. A modified Poisson regression approach to prospective studies with binary data. Am J Epidemiol. 2004; 159:702-706. [PubMed: 15033648]

21. Joffe SW, Webster K, McManus DD, et al. Improved survival after heart failure: a communitybased perspective. J Am Heart Assoc. 2013; 2:e000053. [PubMed: 23676294]

22. Loh JC, Creaser J, Rourke DA, et al. Temporal trends in treatment and outcomes for advanced heart failure with reduced ejection fraction from 1993-2010: findings from a university referral center. Circ Heart Fail. 2013; 6:411-419. [PubMed: 23479563]

23. Cheung AC, Rabie RN, Marquez M, et al. The clinical presentation of decompensated cirrhosis: a comparison of two time periods. Hepatology. 2013; 58:1197A. [PubMed: 23696103]

24. Vergara M, Cleries M, Vela E, et al. Hospital mortality over time in patients with specific complications of cirrhosis. Liver Int. 2013; 33:828-833. [PubMed: 23496284]

25. Runyon BA. Management of adult patients with ascites due to cirrhosis: an update. Hepatology. 2009; 49:2087-2107. [PubMed: 19475696]

26. Bernard B, Grange JD, Khac EN, et al. Antibiotic prophylaxis for the prevention of bacterial infections in cirrhotic patients with gastrointestinal bleeding: a meta-analysis. Hepatology. 1999; 29:1655-1661. [PubMed: 10347104]

27. Saab S, Hernandez JC, Chi AC, et al. Oral antibiotic prophylaxis reduces spontaneous bacterial peritonitis occurrence and improves short-term survival in cirrhosis: a meta-analysis. Am J Gastroenterol. 2009; 104:993-1001. quiz 1002. [PubMed: 19277033]

28. Wong F, Bernardi M, Balk R, et al. Sepsis in cirrhosis: report on the 7th meeting of the International Ascites Club. Gut. 2005; 54:718-725. [PubMed: 15831923]

29. European Association for the Study of the Liver. EASL clinical practice guidelines on the management of ascites, spontaneous bacterial peritonitis, and hep-atorenal syndrome in cirrhosis. J Hepatol. 2010; 53:397-417. [PubMed: 20633946]

30. Salerno F, Gerbes A, Gines P, et al. Diagnosis, prevention and treatment of hepatorenal syndrome in cirrhosis. Gut. 2007; 56:1310-1318. [PubMed: 17389705]

31. Orman ES, Hayashi PH, Bataller R, et al. Paracentesis is associated with reduced mortality in patients hospitalized with cirrhosis and ascites. Clin Gastroenterol Hep-atol. 2014; 12:496-503. e491.

32. Dellinger RP, Levy MM, Rhodes A, et al. Surviving sepsis campaign: international guidelines for management of severe sepsis and septic shock: 2012. Crit Care Med. 2013; 41:580-637. [PubMed: 23353941]

33. Ani C, Farshidpanah S, Bellinghausen Stewart A, et al. Variations in organism-specific severe sepsis mortality in the United States: 1999-2008. Crit Care Med. 2015; 43:65-77. [PubMed: 25230374]

34. Jalan R, Gines P, Olson JC, et al. Acute-on chronic liver failure. J Hepatol. 2012; 57:1336-1348. [PubMed: 22750750]

35. Poonja Z, Brisebois A, van Zanten SV, et al. Patients with cirrhosis and denied liver transplants rarely receive adequate palliative care or appropriate management. Clin Gastroenterol Hepatol. 2014; 12:692-698. [PubMed: 23978345] 
36. Walling AM, Wenger NS. Palliative care and end-stage liver disease. Clin Gastroenterol Hepatol. 2014; 12:699-700. [PubMed: 24246765]

37. Joynt KE, Jha AK. Thirty-day readmissions-truth and consequences. N Engl J Med. 2012; 366:1366-1369. [PubMed: 22455752]

38. van Walraven C, Bennett C, Jennings A, et al. Proportion of hospital readmissions deemed avoidable: a systematic review. CMAJ. 2011; 183:E391-E402. [PubMed: 21444623]

39. Gorodeski EZ, Starling RC, Blackstone EH. Are all readmissions bad readmissions? N Engl J Med. 2010; 363:297-298. [PubMed: 20647209]

40. Volk ML, Tocco RS, Bazick J, et al. Hospital read-missions among patients with decompensated cirrhosis. Am J Gastroenterol. 2012; 107:247-252. [PubMed: 21931378]

41. Lo Re V, Lim JK 3rd, Goetz MB, et al. Validity of diagnostic codes and liver-related laboratory abnormalities to identify hepatic decompensation events in the Veterans Aging Cohort Study. Pharmacoepidemiol Drug Saf. 2011; 20:689-699. [PubMed: 21626605]

42. Lo Re V 3rd, Haynes K, Goldberg D, et al. Validity of diagnostic codes to identify cases of severe acute liver injury in the U.S. Food and Drug Administration's Mini-Sentinel Distributed Database. Pharmacoepidemiol Drug Saf. 2013; 22:861-872. [PubMed: 23801638]

43. Singla A, Hart JL, Li Y, et al. Hospitalization for complications of cirrhosis: does volume matter? J Gastrointest Surg. 2011; 15:330-335. [PubMed: 21108014] 


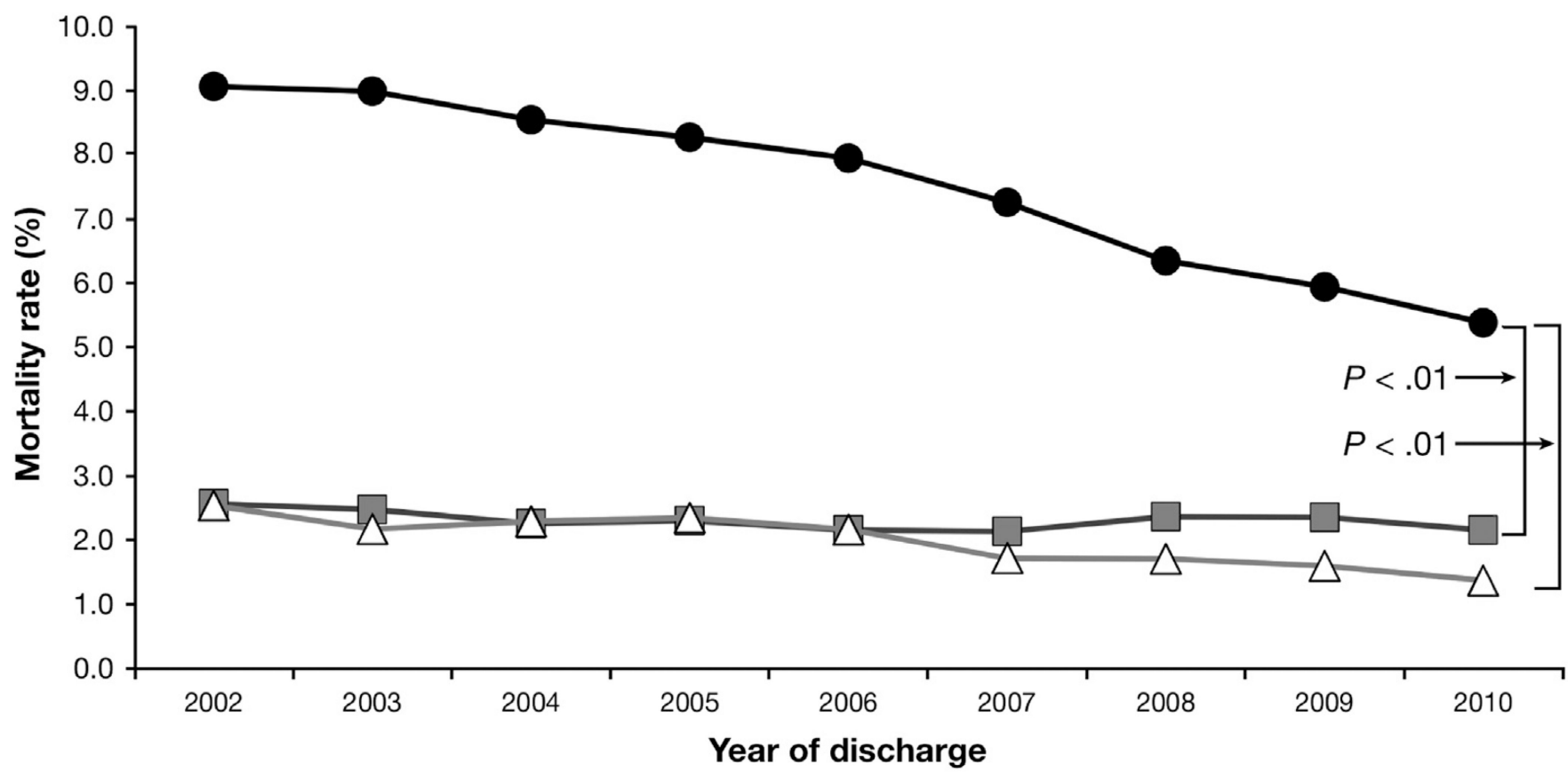

$\rightarrow$ Cirrhotic inpatient mortality $\quad \square-$ Non-cirrhotic (NC) patients $\quad \Delta^{-}$NC, congestive heart failure patients

Figure 1.

Inpatient mortality from 2002 to 2010 for cirrhotic patients, NC patients, and NC patients with CHF. NC and NC-CHF patients were matched 1:1 with cirrhotic patients on age, sex, and year of discharge. The decrease in mortality for patients with cirrhosis was significantly more than that in the NC and NC-CHF cohorts. 


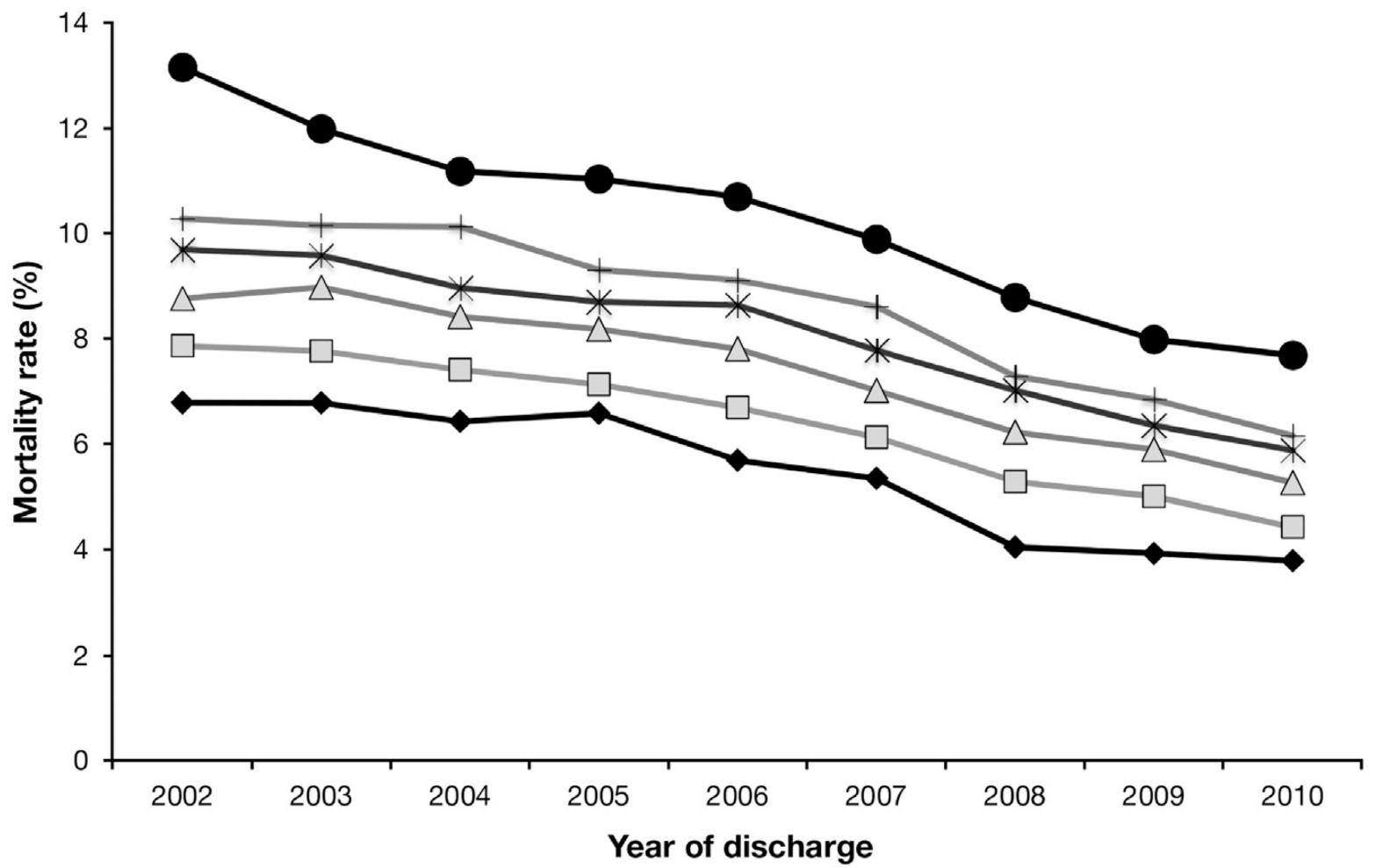

Age group $\leftarrow$ Age $<40 \quad \square$ Age 41-50 $\triangle$ Age 51-60 $\quad *$ Age 61-70 + Age 71-80 $\leadsto$ Age $>80$

Figure 2.

Inpatient mortality from 2002 to 2010 for cirrhotic patients by age group. 


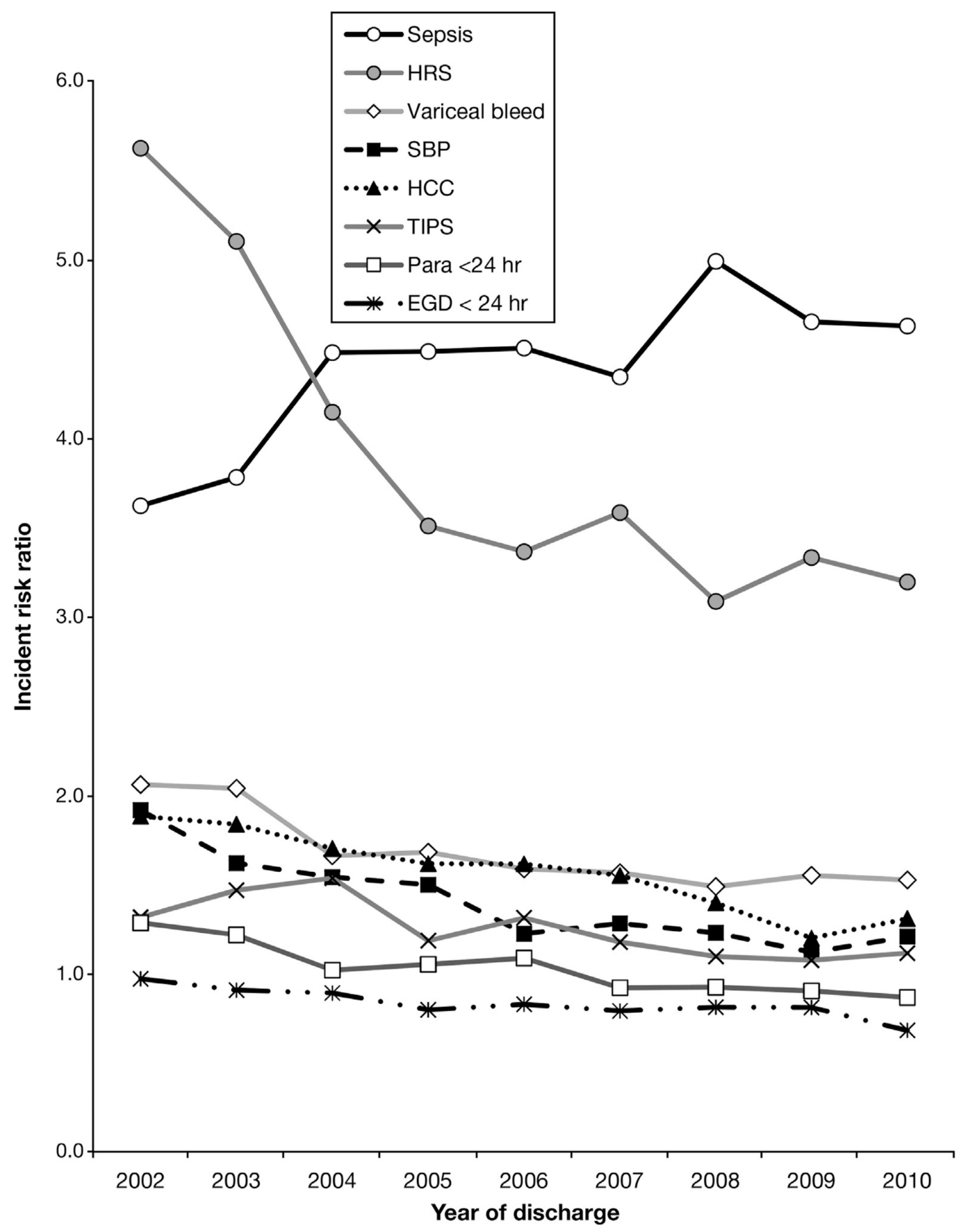

Figure 3.

Incident risk ratios for cirrhosis complications or interventions, each year from 2002 to 2010. Derived from interaction terms (eg, HRS $\times 2002, \times 2003$, to $\times 2010$; sepsis $\times 2002, \times$ 2003, to 2010) in Poisson model for inpatient mortality. (Para = paracentesis). 


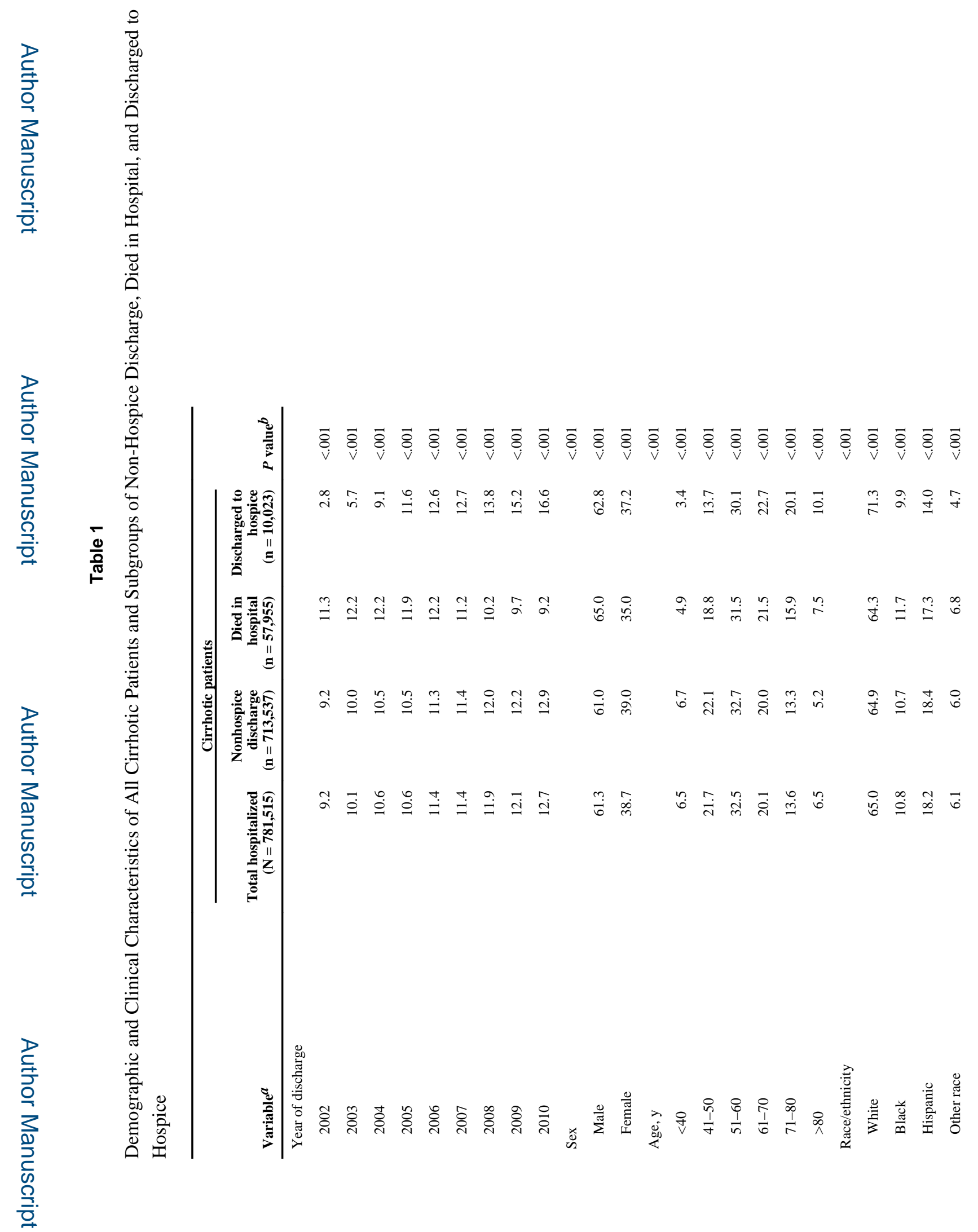




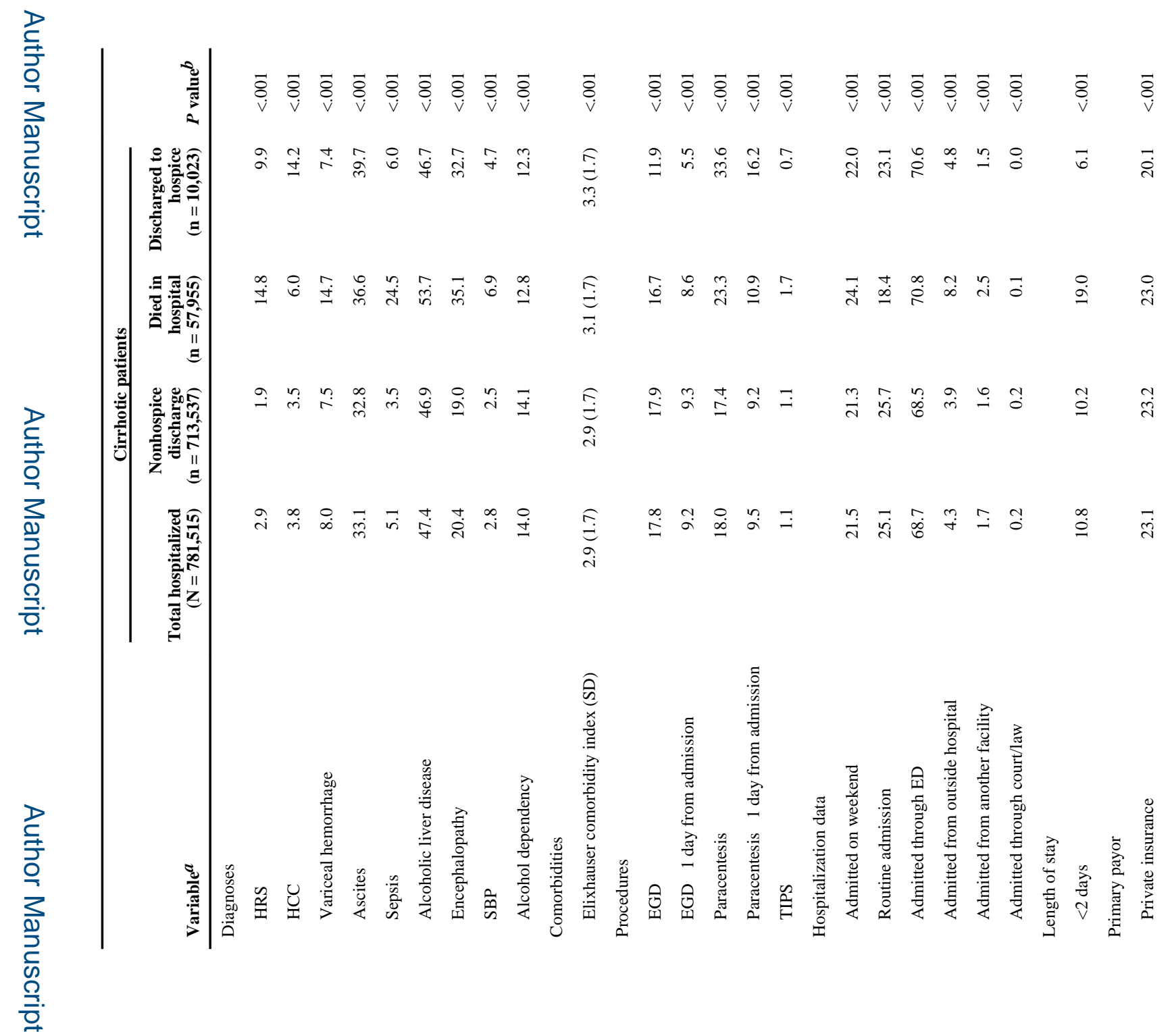
Gastroenterology. Author manuscript; available in PMC 2016 May 01. 


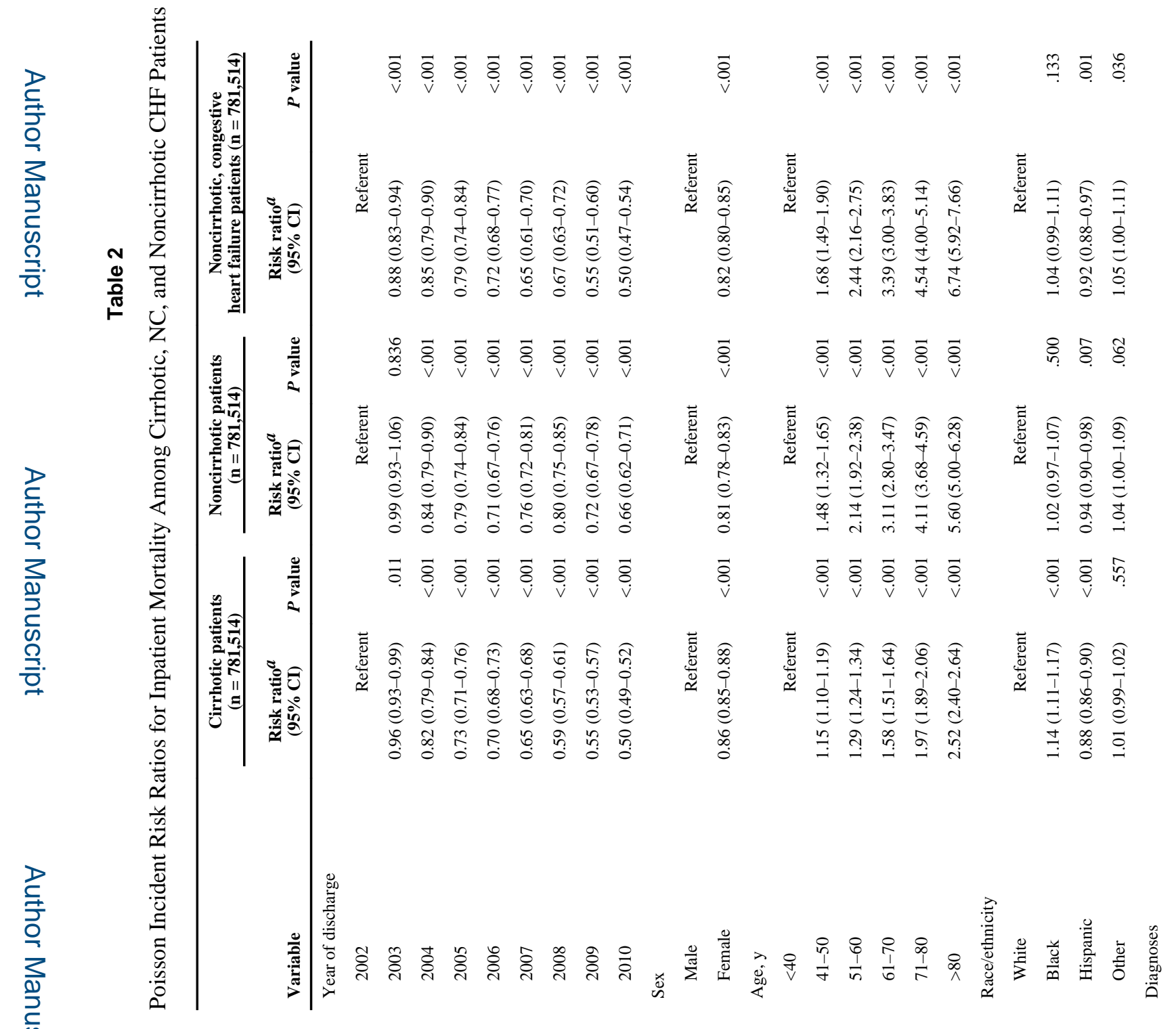




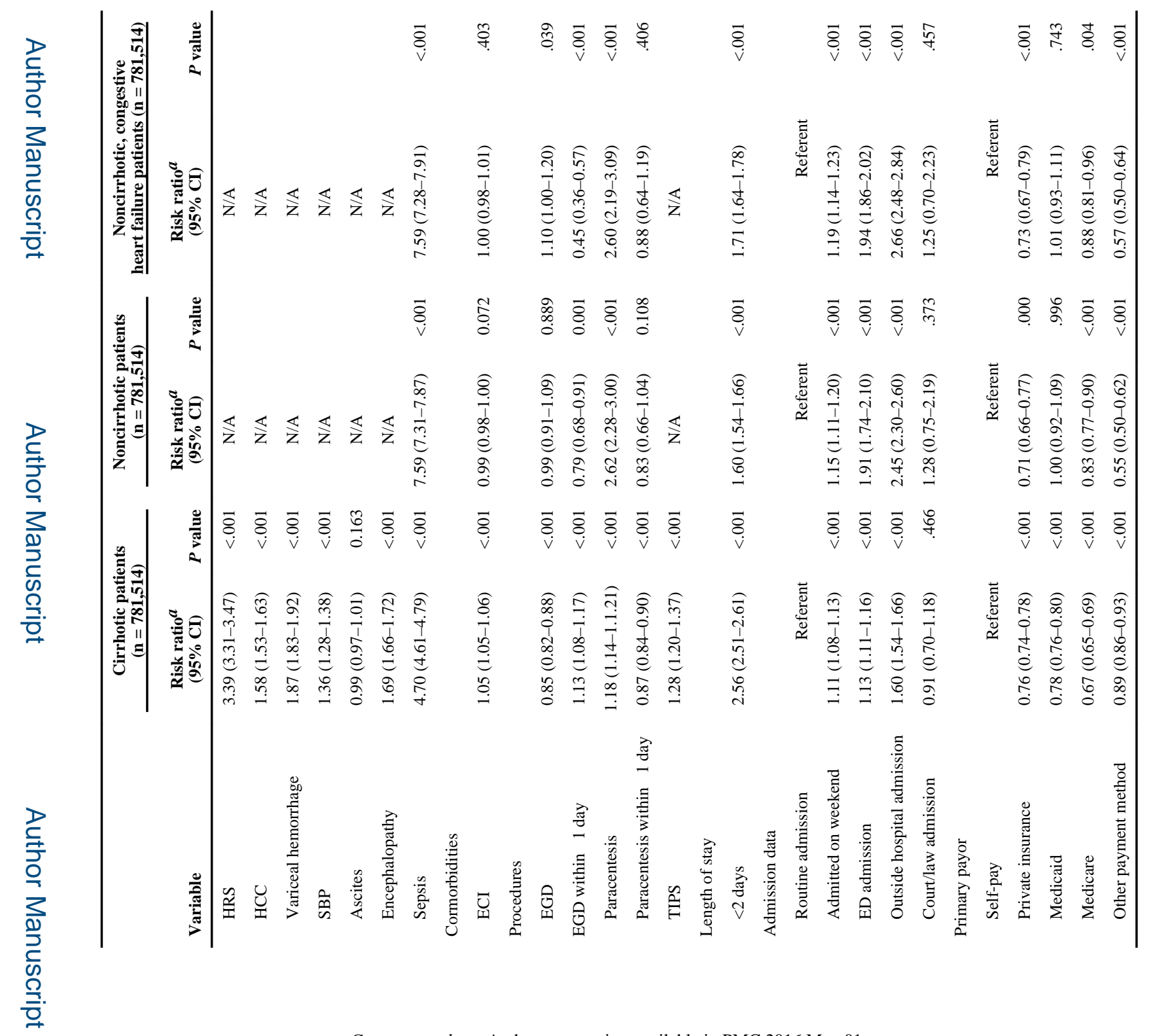
Gastroenterology. Author manuscript; available in PMC 2016 May 01. 

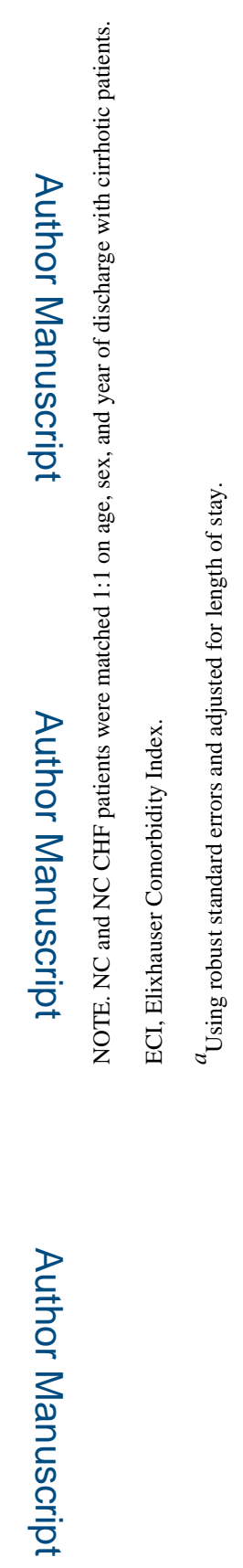

로을

Gastroenterology. Author manuscript; available in PMC 2016 May 01. 
Table 3

Poisson Incident Risk Ratios for Discharge to Hospice Among Cirrhotic Patients

\begin{tabular}{|c|c|c|}
\hline Variable & Risk ratio $^{a}(95 \%$ CI) & $P$ value \\
\hline \multicolumn{3}{|l|}{ Year of discharge } \\
\hline 2002 & Referent & \\
\hline 2003 & $1.94(1.68-2.25)$ & $<.001$ \\
\hline 2004 & $2.85(2.49-3.27)$ & $<.001$ \\
\hline 2005 & $3.49(3.06-3.99)$ & $<.001$ \\
\hline 2006 & $3.60(3.16-4.11)$ & $<.001$ \\
\hline 2007 & $3.46(3.03-3.94)$ & $<.001$ \\
\hline 2008 & $3.37(2.95-3.85)$ & $<.001$ \\
\hline 2009 & $3.49(3.07-3.98)$ & $<.001$ \\
\hline 2010 & $3.60(3.16-4.10)$ & $<.001$ \\
\hline \multicolumn{3}{|l|}{ Sex } \\
\hline Male & Referent & \\
\hline Female & $0.94(0.90-0.98)$ & .002 \\
\hline \multicolumn{3}{|l|}{ Age, y } \\
\hline$<40$ & Referent & \\
\hline $41-50$ & $1.11(0.98-1.24)$ & .096 \\
\hline $51-60$ & $1.44(1.28-1.61)$ & $<.001$ \\
\hline $61-70$ & $1.83(1.63-2.05)$ & $<.001$ \\
\hline $71-80$ & $2.64(2.33-2.98)$ & $<.001$ \\
\hline$>80$ & $3.50(3.07-3.98)$ & $<.001$ \\
\hline \multicolumn{3}{|l|}{ Race/ethnicity } \\
\hline White & Referent & \\
\hline Black & $0.90(0.87-0.97)$ & .007 \\
\hline Hispanic & $0.71(0.67-0.76)$ & $<.001$ \\
\hline \multicolumn{3}{|l|}{ Diagnoses } \\
\hline HRS & $2.28(2.12-2.44)$ & $<.001$ \\
\hline $\mathrm{HCC}$ & $3.49(3.30-3.69)$ & $<.001$ \\
\hline Variceal hemorrhage & $1.15(1.06-1.24)$ & .001 \\
\hline SBP & $1.07(0.97-1.18)$ & .156 \\
\hline Ascites & $1.18(1.12-1.23)$ & $<.001$ \\
\hline Encephalopathy & $1.73(1.66-1.81)$ & $<.001$ \\
\hline Sepsis & $0.91(0.83-0.99)$ & .024 \\
\hline \multicolumn{3}{|l|}{ Comorbidities } \\
\hline Elixhauser Comorbidity Index & $1.05(1.04-1.06)$ & $<.001$ \\
\hline \multicolumn{3}{|l|}{ Procedures } \\
\hline EGD & $0.63(0.58-0.68)$ & $<.001$ \\
\hline EGD $<1$ day from admission & $0.97(0.87-1.09)$ & .649 \\
\hline Paracentesis & $1.91(1.80-2.02)$ & $<.001$ \\
\hline Paracentesis $<1$ day from admission & $0.95(0.89-1.02)$ & .167 \\
\hline
\end{tabular}




\begin{tabular}{lcr}
\hline Variable & Risk ratio $^{\boldsymbol{a}} \mathbf{( 9 5 \%}$ CI) & $\boldsymbol{P}_{\text {value }}$ \\
\hline Thoracentesis & $1.41(1.24-1.60)$ & $<.001$ \\
Thoracentesis $<1$ day from admission & $0.96(0.78-1.17)$ & .655 \\
TIPS & $0.56(0.45-0.71)$ & $<.001$ \\
Length of stay & & \\
$<2$ days & $0.63(0.8-0.68)$ & $<.001$ \\
Admission source/day & & \\
Routine admission & Referent & \\
Admitted on weekend & $1.06(1.01-1.11)$ & .013 \\
ED admission & $0.78(0.75-0.82)$ & $<.001$ \\
Outside hospital admission & $0.71(0.62-0.80)$ & $<.001$ \\
Court/law admission & $0.14(0.03-0.54)$ & .005 \\
Primary payor & & \\
Self-pay & Referent & \\
Private insurance & $0.74(0.68-0.80)$ & $<.001$ \\
Medicaid & $0.80(0.73-0.87)$ & $<.001$ \\
Medicare & $0.77(0.71-0.84)$ & $<.001$ \\
Other payment method & $1.29(1.16-1.44)$ & $<.001$ \\
\hline
\end{tabular}

${ }^{a}$ Using robust standard errors and adjusted for length of stay. 